initiatives in improving search results. In addition, we conducted interviews of web leaders in industry at a pre-selected Google event and summarised the applicable findings.

Results A few core findings were clearly evident as follows: keywords are still important. However search algorithms are being modified to discover meaning beyond a simple query labelled 'semantic search.' 'Authority' is being measured and being a government entity or an entity recognised with a proven record of producing scientifically useful evidence is of real benefit. Regular fresh content is essential and simple language and coherent writing are recognised and rewarded. Finally, and most importantly social media drives search and must be a part of an organisations overall communication strategy.

Conclusion Search engines are famously creative in encouraging breakthroughs that deliver information to searchers and therefore are constantly changing their algorithms. Recognising the importance of these changes and their online effect is essential. The application of the seven cardinal factors to improve our overall SEO strategies will provide us a roadmap for success in engaging our communities.

\section{WIKIPEDIA WORKSHOP FOR HEALTH PROFESSIONALS: A HANDS-ON COURSE FOR EXTENDING THE REACH. ENGAGEMENT AND IMPACT OF YOUR OCCUPATIONAL SAFETY AND HEALTH INFORMATION}

Max R Lum*. National Institute for Occupational Safety and Health, Washington, D.C., USA

\subsection{6/oemed-2018-ICOHabstracts.212}

Aim of Workshop This workshop has been designed as an interactive session with $40 \mathrm{~min}$ of overview followed by a 2 hour hands on period of exercises that assist participants to design, manage, and track the use of edits to Wikipedia for increasing the engagement of wider audiences and extending the reach of $\mathrm{OSH}$ information including research findings, and guidance documents. Participants will gain practical skills concerning security protection, tracking of edits, evaluation, ROI, the culture of Wikipedia and become familiar with easy to use digital tools through online hands-on tutorials. Take away selflearning information will be provided to assist future reference.

${ }^{1}$ Max Lum, ${ }^{2}$ Thais Morata, ${ }^{2}$ Garrett Burnett

${ }^{1}$ National Institute for Occupational Safety and Health, Washington, D.C. USA

${ }^{2}$ National Institute for Occupational Safety and Health, Cincinnati, Ohio. USA

\section{$1674 a$ USING WIKIPEDIA TO BUILD OUR OSH COMMUNITY OF PRACTICE: AN GENERAL OVERVIEW}

Max Lum*, Thais Morata, Garrett Burnett. National Institute for Occupational Safety and Health, Washington DC, USA

\subsection{6/oemed-2018-ICOHabstracts.213}

Introduction The Internet has become the important health information resource for patients, health providers and the general public. Wikipedia a collaboratively written web-based encyclopaedia has become the dominant online reference work. Wikipedia ranks as the eighth most accessed Web site on the internet. Wikipedia is the number one driver (after organic search) of visitors to the NIOSH (USA) web site. This workshop is structured to provide a basic understanding of Wikipedia to familiarise health professionals on how to use Wikipedia to disseminate and track important occupational safety and health information to a large global audience.

Methods The curriculum for this workshop is guided by the results of an investigation of how traffic to Wikipedia articles correlated with traffic to the main selected web sites and how page view statistics compared to organic search and other web site drivers. We compared Wikipedia's occurrence, mean position to other Web sites, and examined which factors influence Web site ranking. In addition, key edit factors, long-tail search results, comparison of edits to premier web pages and other key factors were examined. A key part of this workshop will be the appropriate use of metrics and tracking to guide outcomes and impact.

Results By developing and implementing a carefully crafted online strategy Wikipedia edits have become a major source of driving searchers of occupational safety and health information to the NIOSH Web site.

Conclusion The rank of a Web site among search engines results depends on factors such as the search engine algorithm and search engine optimizations strategies (SEO), which aim to influence rankings. This workshop is structured to address the important factors that need to be a key part of your Wikipedia editing strategy to optimise algorithm mediation of traffic to your information.

\section{CONTRIBUTION OF ICOH TO OSH AT NATIONAL AND INTERNATIONAL LEVEL: DEVELOPMENT OF A SURVEY}

${ }^{1} \mathrm{~V}$ Boccuni*, ${ }^{1} \mathrm{P}$ Dionisi, ${ }^{1} \mathrm{C}$ Petyx,${ }^{2} \mathrm{~A}$ Oliverio, ${ }^{1} \mathrm{BM}$ Rondinone, ${ }^{1} \mathrm{~F}$ Boccuni, ${ }^{1} \mathrm{~A}$ Valenti, ${ }^{1} S$ Iavicoli. 'INAIL, Department of Occupational and Environmental Medicine, Epidemiology and Hygiene, Monteporzio Catone (RM), Italy; ${ }^{2} \mathrm{ICOH}$, International Commission on Occupational Health, Monteporzio Catone (RM), Italy

\subsection{6/oemed-2018-ICOHabstracts. 214}

Introduction The International Commission on Occupational Health (ICOH) is an international professional organisation dedicated to scientific progress, knowledge, and development of occupational health and safety. Founded in 1906 in Milan, Italy, ICOH currently has over 2000 members in 96 countries. The 37 Scientific Committees provide a forum for members and associated experts to meet in order to promote, share and discuss progress relating to the OSH topics. The aim of this study is to develop a survey on ICOH contribution to $\mathrm{OSH}$ at national and international level.

Methods The survey project will consist of the following phases: first draft of the questionnaire shared with ICOH Officers, members of the Task Group on Membership and National Secretaries; second draft based on the suggestions and inputs received; pilot test; questionnaire administration; data elaboration and analysis; dissemination of results.

The questionnaire will be administered to $\mathrm{ICOH}$ members in good standing through the dedicated web-based platform SurveyMonkey. The language selected for the questionnaire will be English.

The questionnaire will be structured along different sections investigating $\mathrm{ICOH}$ contribution to $\mathrm{OSH}$ at national and international level, activities carried out by Scientific Committees and National Secretaries, results visibility and dissemination at International Congresses, effectiveness of ICOH communication tools. Data will be analysed using SPSS Statistics V.22. 
Results and Conclusion Preliminary results will be presented at ICOH 2018 Congress.

This study will be, for the first time in $\mathrm{ICOH}$ history, addressed to the whole active membership.

It might become the starting point for a systematic monitoring survey on needs and perceptions of members with respect to $\mathrm{ICOH}$ commitment in the OSH sector. The outputs will also contribute to the improvement of $\mathrm{ICOH}$ scientific activities and identification of the new OSH priorities at national and international level.

\section{\begin{tabular}{|l|l}
\hline 726 & OUR GRATITUDE AND APPRECIATION FOR YOUR
\end{tabular} FEEDBACK}

${ }^{1} \mathrm{AM}$ Howard, ${ }^{2} \mathrm{C}$ Harrison. ${ }^{1} \mathrm{CNM} 3$, Occupational Health, HSE South East; ${ }^{2}$ Waterford Healing Arts, Artist in Residence, 2017

10.1136/oemed-2018-ICOHabstracts.215

Introduction In May 2017 the Occupational Health Safer Better Care Standards were launched.

Standard 1.4 of the Occupational Health, Safer Better Care Standards states' Workers feedback including complaints and compliments are responded to promptly, openly and effectively with clear communication and support provided throughout this process'

Method On an ongoing basis in Occupational Health we have a suggestion box in our four Occupational Health Departments for feedback.

In our work plan for 2017 we had decided to dedicate the month of May to doing a client satisfaction survey. The survey questionnaire is based on the validated national occupational service patient survey in the National Health Service UK (NHS). We decided as a team that each of the four Occupational Health units in the South East would hand this survey to clients who pass through their departments in the month of May.

In addition Ann Marie Howard, CNM3 asked the Occupational Health staff to send her any Thank you cards they had received. Ann Marie met with Ciara the Artist in residence in Waterford and discussed ideas with her in relation to what we could with the Thank you cards and feedback cards kindly received.

It was decided we would do a calendar for 2018 and call it' Occupational Health- Our Gratitude and Appreciation for your feedback'. We decided to use a theme for each month for example January and July are the months where you have new Junior Doctors, September is a month when you have an intake of student nurses and October is the month for Influenza vaccination. Along with the theme for each month we are taking words, sentence clients wrote in there Thank you cards and Feedback and illustrate them throughout the calendar.

Results The Thank you cards and feedback cards have all been collected. The May 2017 client satisfaction survey has yet to be analysed and results written up. It is a work in progress.

Discussion Our hope would be we will get the calendar published and communicate it to all client users as a feedback mechanism on our gratitude and appreciation.

Following on from this project it would be a wonderful addition if an app could be developed for Occupational Health as a communication tool.
756

'PREDICTED SICKNESS ABSENCE FROM EMPLOYMENT COMPARED WITH DEPARTMENT OF SOCIAL PROTECTION GUIDELINES FOR CLOSED CERTIFICATION WITH COMMON HEALTH PROBLEMS'

${ }^{1} \mathrm{~N}$ Kononenko*, ${ }^{2} \mathrm{E}$ Loftus, ${ }^{2} \mathrm{D}$ Whelan. ${ }^{1} \mathrm{~S} P R$ in Occupational Medicine, CHI, Dublin; ${ }^{2}$ Consultant Occupational Physician, CIE, Dublin

\subsection{6/oemed-2018-ICOHabstracts.216}

Introduction Prolonged absence from work may cause deterioration in the patient's health and wellbeing and threaten job security.

The Department of Social Protection issued Guidelines for Closed Certification in September 2015 to assist medical certifiers of sickness absence.

These Guidelines have been complied for use in an Irish context with regard to evidence-based protocols on various health problems, which were developed by DSP, and also with reference to the Official Disability Guidelines 2013, to The Medical Disability Advisor: Workplace Guidelines for Disability Duration 2009.

Methods This was an observational study. A questionnaire survey emailed to Consultants and HST trainees of different specialties to explore their opinion on predicted duration of sickness absence with different medical conditions and interventions.

71 Consultants and HST trainees from different Medical Specialties and 32 Consultants and HST trainees in Occupational Medicine responded to a survey. The response rate was $29 \%$ from Medical Specialists and 44\% from Occupational Medicine Specialists.

Results According to this survey 60\% of Physicians and 44\% of Occupational Health Physicians are not familial with Closed Certification Guidelines.

Medical specialist's opinion coincides with Guidelines in 4\%-93\%, Occupational Medicine Specialists in 4\%-100\%. The agreement with Guidelines is in responses about less serious (infectious) conditions in both groups, disagreement with Guidelines in responses about more serious medical conditions (cardiac, pneumonia, asthma).

There is a trend to predict shorter time for recovery then recommended by Guidelines in both groups. There is no significant difference in predicted absence agreed with Guidelines recommendations among two groups.

Discussion This study compared the opinion of specialists in predicting duration of sickness absence with durations laid down in 'Closed Certification Guidelines for General Practitioners'

Not all clinician's opinion coincides with recommended by Closed Certification Guidelines.

More than half of participants were not aware about Closed Certification Guidelines.

\section{IWH RESEARCH ALERT - STAYING CURRENT WITH OHS LITERATURE}

Q Mahood*, D Van Eerd, J Liu, E Irvin. Institute for Work and Health, Toronto, Canada

\subsection{6/oemed-2018-ICOHabstracts.217}

Introduction Keeping abreast of the current literature is difficult for any researcher but OHS researchers have particular difficulty because the literature cuts across a variety of fields, such as medicine, public health, psychology, and business. To 